\title{
Variation in the incidence of diverticular disease within the city of Edinburgh
}

\author{
M. A. EASTWOOD, JEAN SANDERSON, S. J. POCOCK, AND W. D. MITCHELL \\ From the Wolfson Gastrointestinal Laboratories, Gastrointestinal Unit, Western General Hospital, \\ Edinburgh, the Medical Computing and Statistics Group, Medical School, University of Edinburgh, \\ Edinburgh, and the Department of Clinical Chemistry, Oldchurch Hospital, Romford, Essex
}

SUMMARY An estimate of the incidence of diverticular disease of the colon was based on all the barium enema examinations in Edinburgh over four years (12 335 cases); using the census data for 1971 , incidence rates for the city by age, sex, and electoral ward were calculated. The overall annual incidence rate was $1 \cdot 55 / 1000$, the rate rising sharply with increasing age. The incidence of diverticular disease varies from ward to ward (range 0.92-2.04/1000), adjacent wards have similar incidence rates and the six wards with the lowest rates are clustered together in the South East of the city. Ward incidence rates have a significant negative association with the percentage of owner occupiers living in that area.

While it has been suggested that diverticular disease of the colon is a very common condition (Painter, 1975) there have been very few studies in Britain on the incidence or prevalence of diverticular disease. Methods for identifying diverticular disease which have been principally used are necropsy (Parks, 1968) and barium studies (Manousos et al., 1967), each of which are selective in the choice of patientthat is, the death or presentation for barium enema will each produce its own peculiar bias. Diverticular disease affects a substantial proportion of the older age groups among Western populations so that the prevalence rate may amount to more than $30 \%$ of persons over the age of 60 (Dearlove, 1954; Hughes, 1969; Havia, 1971). This paper describes the distribution of new cases of diverticular disease through the City of Edinburgh based on the reporting of barium enema examinations.

\section{Methods}

All barium enema examinations of the colon for the City of Edinburgh during the period 1970-73 inclusive were noted. For all individuals with barium enema showing diverticular disease the age, sex, and electoral ward in which they lived were identified.

The census population figures recorded in the 1971 census were used so that incidence rates could

Received for publication 2 December 1976 be calculated. Further census data also enabled certain social characteristics and geographical locations to be studied as possible factors affecting incidence rates.

\section{Results}

During the period 1970-73 there were 12335 barium enemas conducted on Edinburgh residents at six different hospitals in the City; 2193 (22.9\%) were diagnosed as diverticular disease, with the number of cases diagnosed each year being similar. The percentages with positive diagnoses in the six hospitals were also similar, ranging from $20.7 \%$ to $26.4 \%$. There were 12 instances of duplicate tests on the same individual (or possibly a close relative at the same address with the same name), in which case it seems sensible to conclude that there were 2181 new cases of diverticular disease.

The population of Edinburgh over the age of 15 years was recorded in the 1971 census as 352603 . Hence on the basis of the positive enemas the calculated annual incidence of diverticular disease is $1 \cdot 55 / 1000$ adult population. The Table shows the pattern of incidence by age and sex. There is the anticipated sharp rise in incidence rate with increasing age. In each group the female rate is slightly lower than the male rate. However, because of the greater numbers of older females, the overall crude incidence rate for females is slightly higher 
Table Annual incidence rates of diverticulitis by age and sex (per 1000)

\begin{tabular}{llll}
\hline Age group $(y r)$ & Males & Females & Total \\
\hline $15-44$ & 0.18 & 0.15 & 0.17 \\
$45-59$ & 1.36 & 1.26 & 1.30 \\
$60-74$ & 3.97 & 3.82 & 3.88 \\
$75+$ & 6.24 & 5.55 & 5.74 \\
Total & 1.39 & 1.68 & 1.55 \\
\hline
\end{tabular}

than for males. In fact, the sex difference is not statistically significant after allowing for age and electoral ward effects. Since the 23 electoral wards in Edinburgh have different age and sex distributions, allowance has to be made for age and sex in assessing the incidence rates by ward. This has been obtained using a linear-logistic model for the effects of ward, age and sex on incidence rates. Figure 1 shows a geographical distribution of wards in Edinburgh with the ward incidence standardised for age and sex and also the ranking of the ward. There was considerable variation in annual incidence rates (range 0.92-2.04/1000) (Figs. 1 and 2). Indeed there was much more variation between wards than can be achieved by chance. A likelihood ratio-test gave a highly significant result $\left(\chi^{2}=70 \cdot 17\right.$ on 22 degrees of freedom $P<0.0001)$. There are two striking features of this geographical distribution of incidence ranks. The two wards with the highest incidence, wards 1 and 2 , are adjacent in the centre of the city and the six wards with the lowest incidence are clustered in the south and east of the city. The significance of such clustering can be tested by a spacial autocorrelation coefficient (Cliff, 1973) which equals $+0.292 \quad(P<0.02)$, suggesting that the observed clustering is unlikely to have arisen by

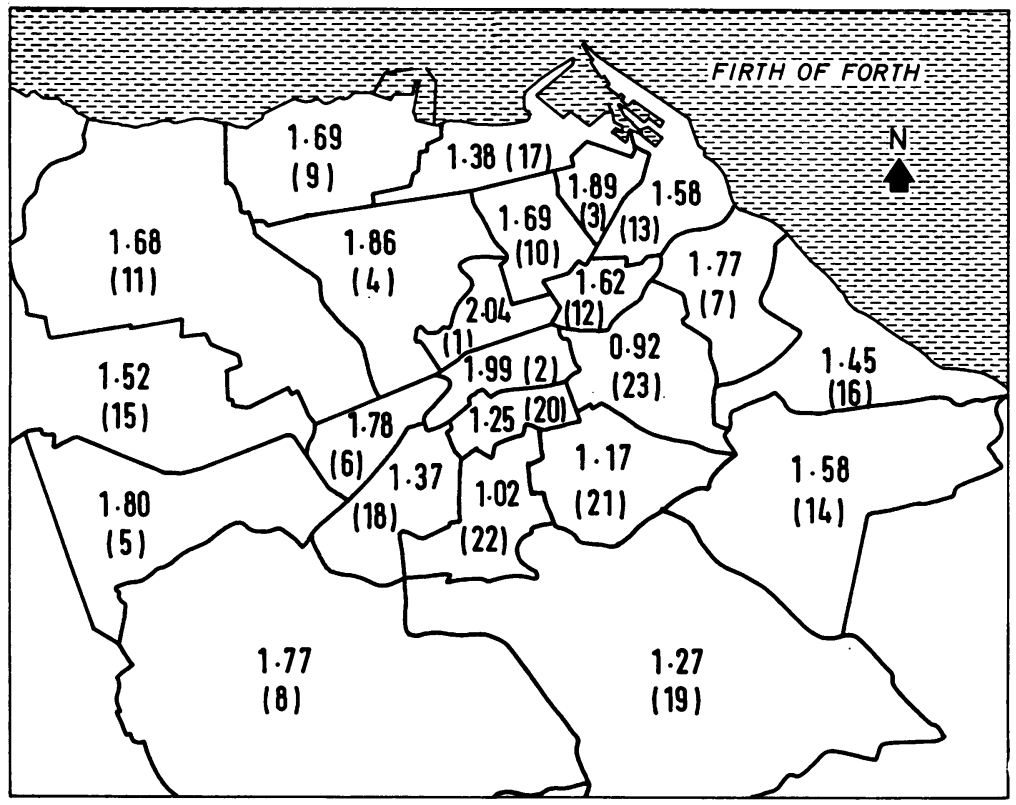

Fig. 1 A map of Electoral wards in Edinburgh showing the annual incidence rate 1000 adult population (diverticular disease) and the rank-highest to lowest $(1-23)$ in parentheses.

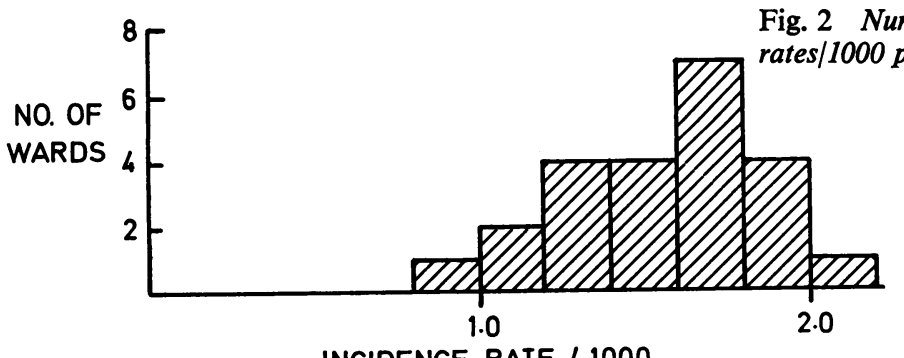

Fig. 2 Number of wards with equivalent annual incidence rates/1000 population of diverticular disease.

INCIDENCE RATE / 1000 
chance. Figure 2 shows the incidence rates which when graphed in a histogram appear somewhat skew to the left with low incidence wards being further away from the median $1 \cdot 62 / 1000$ than the high incidence wards.

Information by ward on social class was obtained from the 1971 census. The mean social class of each ward was calculated using the obvious scoring from $1-5$, and this mean ranged in value from 2.42 for ward 22 to 3.56 for ward 2. The rank correlation coefficient between mean social class and incidence rate is +0.19 but is not significantly different from zero $(P=1 \cdot 19)$, so that there is insufficient evidence to establish a definite association. However, it does appear as though wards with a high mean social class - that is, working class areas - tend to have a higher incidence, with one exception, ward 23, which has a low incidence and is predominantly working class. Another social measure for each ward is the percentage of residents who are owner/ occupiers. There is some suggestion that wards with a high percentage of owner/occupiers tend to have a lower incidence rate. The rank correlation coefficient is -0.29 which is significantly different from zero at the $5 \%$ level. Other social measures studied were percentages of houses with the three basic amenities, a car, and more than one person per room but none of these showed any association with incidence rates.

One possibility was that the difference in ward incidence rates might reflect differences in the availability of medical practitioners. Hence, the number of adults per general practitioner was calculated for each ward and compared with the ward incidence rate, the rank correlation being $0.15(P=0.30)$ which is not significant.

\section{Discussion}

Recent theories on the aetiology of diverticular disease suggest that an absence of vegetable dietary fibre or, more specifically, cereal bran from the diet for many years results in the genesis of diverticular disease (Painter, 1975). One might have expected that such inadequacies in dietary habits would apply to all sections of the population so that the incidence of such a slowly evolving condition would be fairly uniformly spread throughout any defined urban area. However, the examination of incidence rates in the electoral wards of Edinburgh during 1970-73 has revealed considerable variations regarding the geographical distribution of diverticular disease with annual ward incidence rates ranging from 0.92 to 2.04 per 1000 adult population.

The identification of diverticular disease by barium enema examination after the patient has been seen by the family doctor and thereafter hospital con- sultants implies that a somewhat complicated selection process might operate, though it is not possible to show any difference in medical practice throughout Edinburgh which might explain such variation. For example, the rate of positive diagnosis made in the various hospitals was approximately the sameround about $23 \%$ and there was no association between ward incidence and the availability of general practitioners. Incidence rates were adjusted for ward differences in age and sex so that these factors can also be discounted. Actually, the apparently higher incidence rates for females can be entirely explained by the excess of older females in the population so that there is no genuine imbalance in disease incidence between the sexes.

One striking result is the similarity in the incidence rate for adjacent wards, particularly the highest two which are both in the older part of the city but remain socially somewhat distinct. The one is largely professional class dwellings and the other contains a very high proportion of social class IV and $\mathrm{V}$ living in lodging houses. The significant inverse relationship between percentage owner/ occupier and ward incidence rate is interesting, though the corresponding association between mean social class and incidence rates were not quite as definitive. These results suggest the possibility that social differences in the quality of diet could account for some of the variations in disease incidence. There are, of course, demonstrations that racial groups have wide variations in the prevalence of diverticular disease (Kyle et al., 1967; Painter, 1975) but as far as we know there is no evidence of a study similar to this present report. Manoussos (1967) suggests that the prevalence of diverticular disease was $7.6 \%$ under the age of 60 years but $35 \%$ over the age of 60 years in a population drawn from apparently healthy volunteers. If this is the case, with our overall incidence rate of patients presenting to hospital of $1.55 / 1000$ population, then diverticular disease must be a somewhat benign condition. It has been shown that the socioeconomic status of patients with colonic cancer appears to be somewhat higher than that of patients with rectal carcinoma (Lynch et al., 1976) and that city dwellers have a higher risk of developing colorectal carcinoma than rural dwellers (Haenszel and Dawson, 1965). There have been no studies similar to the present study for diverticular disease, though there have been shown to be differences in hospital admission rates for diverticular disease throughout Britain (Eastwood et al., 1974).

Overall, this study would seem to indicate that there are some social or geographical factors affecting the presence of diverticular disease within this particular city. It is possible, however, that some 
factors may influence more the presentation of symptoms or the seeking of medical advice rather than actual genesis of diverticulae. There would seem a need for more detailed epidemiological studies of diverticular disease. It would be difficult to carry out any prospective studies for such a slowly evolving condition, so that a retrospective comparison of newly identified cases with controls matched for age might be the most informative approach.

Lastly, it is well known that in Edinburgh, as in other Scottish cities, there is a rather low consumption of vegetable fibre in the population (Ministry of Agriculture, Fisheries, and Food, 1973, 1974). This might imply a rather higher incidence of diverticular disease than in other parts of the United Kingdom. It would be interesting if data were available to make inter-city comparisons of the incidence of positive barium enemas.

We wish to acknowledge the financial assistance given by the Incorporated National Association of British and Irish Millers Limited. We are also grateful to the consultant radiologists in the Edinburgh hospitals who allowed access to their department.

\section{References}

Cliff, A. D. (1974). Spatial Autocorrelation, Academic Press: London.

Dearlove, T. P. (1954). Diverticulitis and diverticulosis with a report on a rare complication. Medical Journal of Australia, 1, 470-475.

Eastwood, M. A., Fisher, N., Greenwood, C. T., and Hutchinson, J. B. (1974). Perspectives on the bran hypothesis. Lancet, 1, 1029-1033.

Havia, T. (1971). Diverticulosis of the colon. Acta Chirurgica Scandinavica, 137, 367-373.

Haenszel, W., and Dawson, E. A. (1965). A note on mortality from cancer of the colon and rectum in the United States. Cancer, 18, 265-272.

Hughes, L. E. (1969). Post mortem survey of diverticular disease of the colon. Gut, 10, 336-351.

Kyle, J., Adesola, A. O., Tinckler, L. F., De Beaux, J. (1967). Incidence of diverticulitis. Scandinavian Journal of Gastroenterology, 2, 77-80.

Lynch, H. T., Guirgis, H., Lynch, J., Brodkey, F. D., and Magee, H. (1975), Cancer of the colon; socio-economic variables in a community. American Journal of Epidemiology, 102, 119-127.

Manoussos, O. N., Truelove, S. C., and Lumsden, K. (1967). Prevalence of colonic diverticulosis in the general population of Oxford area. British Medical Journal, 3, 762-763.

Ministry of Agriculture, Fisheries and Food (1973, 1974). Household Food Consumption and Expenditure 1970 and 1972. HMSO: London.

Painter, N. S. (1975). Diverticular Disease of the Colon. Heinemann: London.

Parks, T. G. (1968). Postmortem studies on the colon, with special reference to diverticular disease. Proceedings of the Royal Soicety of Medicine, 61, 932-934. 